\title{
Weak Matching Rules for Quasicrystals
}

\author{
Joshua E. S. Socolar \\ Department of Physics, Harvard University, Cambridge, MA 02138, USA
}

\begin{abstract}
Weak matching rules for a quasicrystalline tiling are local rules that ensure that fluctuations in "perp-space" are uniformly bounded. It is shown here that weak matching rules exist for $N$-fold symmetric tilings, where $N$ is any integer not divisible by four. The result suggests that, contrary to previous indications, quasicrystalline ground states are not confined to those symmetries for which the incommensurate ratios of wavevectors are quadratic irrationals. An explicit method of constructing weak matching rules for $N$-fold symmetric tilings in two dimensions is presented. It is shown that the generalization of the construction yields weak matching rules in the case of icosahedral symmetry as well.
\end{abstract}

\section{Introduction}

The discovery of quasicrystalline phases of certain alloys raises a fundamental question in the theory of solid structure. Icosahedral and decagonal quasicrystals have been observed, as have octagonal and dodecagonal samples with a lesser degree of translational order, but no other noncrystallographic symmetries have been observed to date. Is there some a priori principle that prohibits them?

A useful way to approach the question is to represent quasicrystals as spacefilling arrangements of rigid tiles of two or more types, where it is assumed that a good approximation to the actual atomic structure is obtained by decorating each tile of a given type with the same atomic motif. It is well known that quasicrystalline tilings of arbitrary symmetry can be constructed using a variety of algorithms [1]. The mere existence of a tiling does not imply, however, that it is a plausible template for a real atomic structure. It may be that certain tilings or even entire symmetry classes can be eliminated from consideration on physical grounds.

One criterion for physical relevance is locality. In real solids, the interaction between atoms decays rapidly with their separation, so a physically plausible model Hamiltonian should not contain terms depending on the relative positions of widely separated units. In a tiling model, where the energetics are encoded in 
rules determining which configurations of tiles are allowed to occur, the rules must be local in the sense that the configurations specified are of bounded size. One is immediately led to ask whether tilings of a given symmetry exist with the property that their long-range translational order can be ensured by local rules. A negative answer would imply that ordered quasicrystals of that symmetry could not exist in the absence of long-range interactions. If, on the other hand, there do exist local rules which single out a class of ordered tilings, then one might expect to observe real quasicrystals of that symmetry.

Tilings that support "perfect matching rules," which ensure that any infinite tiling is a perfectly ordered quasicrystal of a particular local isomorphism class, are known to exist for the cases of 5- and 10-fold symmetry [2,3], 8- and 12-fold symmetry [4], and icosahedral symmetry [5, 6]. The fact that the incommensurate ratios associated with these symmetries are all quadratic has been emphasized by Levitov in ref. [7], where the question of which 2D tilings support local rules is explicitly addressed. In the course of demonstrating why quadratic irrationals are special for 2D tilings, Levitov found it useful to introduce two less stringent types of matching rules, which he called "strong local rules" and "weak local rules." Strong local rules ensure perfect quasicrystalline order, but the tilings consistent with the rules are not all locally isomorphic. Weak local rules allow bounded deviations from perfect quasicrystalline order (in a sense that will be made precise in the next section). Levitov showed that undecorated $N$-fold symmetric rhombic tilings obtained by the canonical projection technique (see below) do not support strong local rules for any $N$ other than $5,8,10$, or 12 (or the crystallographic cases $2,3,4$, and 6). He also showed that all 5 -fold tilings obtained by canonical projection do support weak local rules. (For a review of the theory of matching rules for 2D quasicrystals, see ref. [8].)

In this paper, it is shown that there exist 2D tilings with $N$-fold symmetry that support weak local rules if $N$ is any odd number or twice an odd number. The result does not contradict those of Levitov, but still comes as somewhat of a surprise. One might have expected that the proven lack of strong matching rules could be shown to imply the impossibility of having weak matching rules, but here we have explicit counterexamples.

The strategy followed is to define a certain constraint on the configurations of tiles in a rhombic tiling and then show that the constraint (called the "alternation condition") has three properties when applied to $N$-fold symmetric tilings with prime $N$ :

1. it is consistent with some space-filling tilings of the plane,

2. it ensures that fluctuations away from perfect quasicrystalline order are uniformly bounded, and

3. it can be implemented using local rules for how tiles join.

The extensions to factorable $N$ and to icosahedral symmetry follow easily from the analysis of prime $N$.

The main purpose of this paper is to present rigorous proofs of the three properties of the alternation condition and thereby elucidate certain features of tilings with $N$-fold symmetry. A significant byproduct of the analysis is that it yields new insights into two classes of tilings that have already appeared in the 
literature: the "generalized Penrose tilings," here called "5-fold canonical projections," composed of rhombi decorated as in [9] and the icosahedral tilings composed of rhombohedra decorated as in [1, p. 22].

Unfortunately, the constructions involved in the proofs are difficult to describe without the aid of several new terms and the introduction of new notation. These definitions of terms and notations have been collected in a list that constitutes Sect. 2. Section 3 contains several lemmas and corollaries that are used in Sect. 4, where the main results are proven. In Sects. 3 and 4, attention is restricted to prime values of $N$. In Sect. 5, the construction of a decoration scheme that enforces the weak matching rules for general prime $N$ is presented. In Sect. 6 the special case of 7 -fold symmetry is examined in more detail. In Sect. 7 non-prime values of $N$ are discussed, in Sect. 8 icosahedral tilings, and Sect. 9 contains some concluding remarks on the implications of the work.

The results presented in this paper supersede the discussion of weak matching rules in ref. [8].

\section{Definitions and Notation}

For the analysis presented in this paper, it is natural to divide the cases of $N$-fold symmetry into five categories: (1) $N$ is prime; (2) $N$ is odd but factorable; (3) $N$ is twice a prime; (4) $N / 2$ is odd but factorable; and (5) $N$ is divisible by four. In the constructions that will be considered for producing tilings of these symmetries, tilings with even values of $N$ appear only as special cases of tilings with $N / 2$-fold symmetry. Furthermore, most of the analysis is not applicable to tilings in category (5). It is therefore adequate to make definitions that are only relevant for tilings in categories (1) and (2), even where natural generalizations exist. In the following definitions and throughout this paper $N$ represents any integer greater than two, $Q$ represents any odd integer greater than two, and $P$ represents any prime number greater than two.

Many of the following definitions are based on concepts that have already appeared in the literature and are repeated here only to establish notation. Further discussion of duals of $\mathrm{N}$-grids and of the projection technique for generating quasicrystal tilings can be found in ref. [1] and the papers and references contained therein. The method of lifting a tiling into a higher dimensional cubic lattice is discussed by Elser [10] and Henley [11]. The concept of weak matching rules was first introduced by Levitov [7].

\subsection{Duals of $N$-Grids}

A grid is a set of non-intersecting infinite grid lines for which every point on the plane lies either on a grid line or between two grid lines. A grid line need not be straight. Associated with every grid is a vector called a star vector. The grid lines are indexed by consecutive integers ranging from $-\infty$ to $+\infty$.

An $N$-grid is a set of $N$ grids in which each grid line in each grid intersects every grid line in every other grid in at most one point, which is not a point of tangency. The star vectors of the grids composing an $N$-grid are denoted $\mathbf{e}_{n}$, with $n=0, \ldots$, 
$N-1$. No two star vectors can be exactly parallel or antiparallel. The indices of the grid lines in the grid with star vector $\mathbf{e}_{n}$ are denoted $k_{n}$.

The vector obtained by rotating a star vector $\mathbf{e}_{n}$ through $90^{\circ}$ in the counterclockwise direction will be denoted $\mathbf{e}_{n}^{*}$.

A symmetric $Q$-grid is a $Q$-grid with star vectors of the form $\mathbf{e}_{n}=(\cos (2 \pi n / Q)$, $\sin (2 \pi n / Q))$. Note that the $Q$-grid itself does not necessarily have $Q$-fold symmetry; only the star vectors do.

A $Q$-fold star is simply the set of star vectors of a symmetric $Q$-grid.

A periodic $Q$-grid is a $Q$-grid in which each grid is composed of equally spaced straight lines normal to its star vector, with the spacing being the same for all grids. In this paper, the periodic $Q$-grids discussed will always be symmetric $Q$-grids as well, so that the equations of the grid lines can be written

$$
\mathbf{x}_{n, k} \cdot \mathbf{e}_{n}=T\left(k+\gamma_{n}\right),
$$

where $\mathbf{x}_{n, k}$ is a point on the $k^{\text {th }}$ grid line perpendicular to the star vector $\mathbf{e}_{n}, T$ is the spacing between grid lines, and $\gamma_{n}$ is a real number called the phase of the grid. Note that only the fractional part of $\gamma_{n}$ is significant.

The dual of an $N$-grid is a tiling composed of parallelograms with vertices, $v$, at positions $\mathbf{w}^{(1)}(v)$ determined as follows. (The reason for using " $\mathrm{w}^{(1)}(v)$ " to represent the position of a vertex will become clear in the next subsection.) The points in the plane that do not lie on any grid line are divided by the $N$-grid into connected open regions. For each $n$, there exists some $k_{n}$ for which the points in a given open region lie between grid lines $k_{n}$ and $k_{n}+1$. The vertex dual to the open region lies at the point $\mathbf{w}^{(1)}=\sum_{n=0}^{N-1} k_{n} \mathbf{e}_{n}$. Note that vertices dual to open regions sharing a common border along a grid line with star vector $\mathbf{e}_{n}$ differ in position by the vector $\mathbf{e}_{n}$.

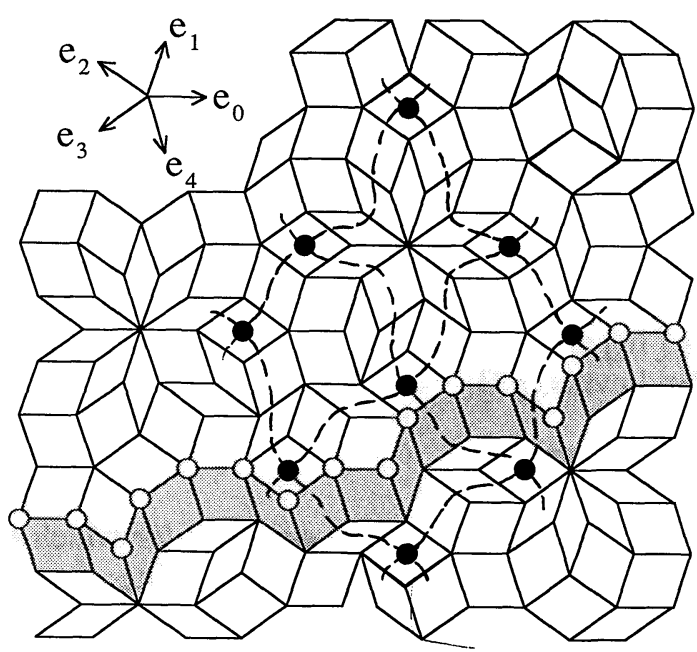

Fig. 1. Definition of tile type, row, row border and net. The star vectors are shown in the inset. Each tile marked with a dot is a (2,3)-tile. (Its edges are parallel to $\mathbf{e}_{2}$ or $\mathbf{e}_{3}$, so it is the dual of an intersection of grid lines with those star vectors.) The shaded tiles form a 4-row. The vertices marked with open circles form a 4-row border. The tiles marked with a dot form a $3 \times 3(2,3)$-net. The curvy lines are guides to the eye, representing the grid lines defining the net 
The dual of an intersection of two grid lines is a parallelogram tile with edges given by the star vectors of the two grids. Every space-filling tiling composed of parallelograms (aligned edge-to-edge) whose edges are taken from a finite set of star vectors, no two of which are parallel, is the dual of some $N$-grid.

The dual of an intersection of grid lines with star vectors $\mathbf{e}_{i}$ and $\mathbf{e}_{j}$ will be referred to as a tile of type $(i, j)$, or simply an $(i, j)$-tile. For a symmetric $Q$-grid, an $(i, j)$-tile is a rhombus with a pair of $|2 \pi(i-j) / Q|$ angles (see Fig. 1).

All tiles having a pair of $|2 \pi k / Q|$ angles are tiles of the same shape but are not necessarily of the same type. Tiles of the same shape are called $k$-tiles. An $(i, j)$-tile is a special case of a $k$-tile if $|i-j|=k$.

The dual of a symmetric $Q$-grid is called a $Q$-fold tiling. It consists of rhombi with each edge parallel to one of the vectors in a $Q$-fold star.

A row is an infinite set of tiles consisting of the duals of all the intersections lying on a single grid line. If the grid line has star vector $\mathbf{e}_{n}$, the row consists of tiles joined along edges parallel to $\mathbf{e}_{n}$ and is referred to as row of type $n$ or an $n$-row (see Fig. 1). Note that a row cannot end in the interior of a portion of tiling composed solely of parallelogram tiles.

A row border is a set of vertices lying on one border of a row (see Fig. 1). If the tile edges in the interior of the row of type $n$ are thought of as instances of the vector $\mathbf{e}_{n}$, the heads of all these vectors form a type $n$ row border (or an $n$-row border), as do the tails.

A $L \times L(n, m)$-net is a set of $(n, m)$-tiles dual to the intersections of $L$ grid lines of type $n$ with $L$ grid lines of type $m$. (An example is shown in Fig. 1.)

\subsection{Lifts, Projections, and Perp-Space Coordinates}

The lift of a $Q$-fold tiling is a unique subset of points of a $Q$-dimensional hypercubic lattice $H$ defined as follows. Let the basis vectors of $H$ be denoted $\mathbf{B}_{n}$. The lift of the vertex at $\mathbf{w}^{(1)}=\sum_{n=0}^{Q-1} k_{n} \mathbf{e}_{n}$ is the hypercubic lattice point $\mathbf{W}=\sum_{n=0}^{Q-1} k_{n} \mathbf{B}_{n}$ and the lift of the tiling is the set of all lifted vertices. The $Q$-dimensional space spanned by the vectors $\mathbf{B}_{n}$ is denoted $E$.

The canonical projection procedure is defined as follows (recall that $Q$ is odd): - $Q \times Q$ projection matrices $\mathscr{P}^{(i)}$ are defined with elements $\mathscr{P}_{j k}^{(i)} \equiv(2 / Q)$ $\cos \left(\frac{2 \pi(j-k) i}{Q}\right)$ for $i=1, \ldots,(Q-1) / 2$ and $\mathscr{P}_{j k}^{(0)} \equiv(1 / Q) \forall j, k$.

- $\mathbf{E}_{n}^{(i)}$ is a $Q$-dimensional vector defined by $\mathbf{E}_{n}^{(i)} \equiv \mathscr{P}_{j k}^{(i)}\left(\mathbf{B}_{n}\right)_{k}$ for $i=1, \ldots,(Q-1) / 2$, $n=0, \ldots, Q-1$. Similarly, $\mathbf{E}^{(0)} \equiv \mathscr{P}_{j k}^{(0)}\left(\mathbf{B}_{n}\right)_{k}$ (the value of $n$ does not matter).

- It is straightforward to show the following:

O For each $i>0$, the $\mathbf{E}_{n}^{(i)}$ span a 2D subspace of $E$, which will be denoted $E^{(i)}$. The 1D subspace spanned by $\mathbf{E}^{(0)}$ will be denoted $E^{(0)}$.

$\bigcirc \mathscr{P}^{(i)} \cdot \mathbf{R}$ is the orthogonal projection of the point $\mathbf{R} \in E$ onto $E^{(i)}$ (including $i=0$ ).

$\circ E^{(i)}$ and $E^{(j)}$ are orthogonal for any pair $i \neq j$.

- $\mathscr{P}^{\perp}$ is defined as $\sum_{i \neq 1} \mathscr{P}^{(i)}=\mathscr{I}-\mathscr{P}^{(1)}$; where $\mathscr{I}$ is the identity. Also define $S(\mathbf{R})$, a unit hypercube, as $\left\{\mathbf{X} \mid 0 \leqq(\mathbf{X}-\mathbf{R}) \cdot \mathbf{B}_{n}<1 \forall n\right\}$, where $\mathbf{R}$ is an arbitrary vector in $E$. $S^{\perp}(\mathbf{R})$ is the image of $S(\mathbf{R})$ under $\mathscr{P}^{\perp}$. 
A canonical projection is a tiling obtained by projecting with $\mathscr{P}^{(1)}$ all points in the lattice $H$ whose images under $\mathscr{P}^{\perp}$ lie in $S^{\perp}(\mathbf{R})$ for some fixed vector $\mathbf{R}$. It can be shown that the procedure for obtaining a canonical projection is completely equivalent to forming the dual of a periodic $Q$-grid [12].

The 2D vectors $\mathbf{w}^{(i)}$ and the scalar $\Gamma$ are defined as follows: We assign to each vertex $v$ the vectors $\mathbf{w}^{(i)}(v) \equiv \sum_{n=0}^{Q-1} k_{n} \mathbf{e}_{n \times i}$, where $i=1, \ldots,(Q-1) / 2, “ \times ”$ denotes the ordinary product, and it is understood here and throughout the paper that the subscripts are taken modulo $Q$. Note that $\mathbf{w}^{(1)}(v)$ is just the ordinary position of the vertex. For different values of $i$, the vectors $\mathbf{w}^{(i)}$ should be thought of as lying in different spaces. We also assign to $v$ an integer $\Gamma(v) \equiv \sum_{n=0}^{Q-1} k_{n}$.

It is straightforward to show that if orthogonal $x$ and $y$ axes in $E^{(i)}$ are defined with the $x$ axis parallel to $\mathscr{P}^{(i)} \mathbf{B}_{0}$, then $\mathbf{w}^{(i)}(v)$ is the same as the projection of the lift of $v$ onto $E^{(i)}$, up to an overall scale factor. Similarly, $\Gamma(v)$ species the projection of the lift of $v$ onto $E^{(0)}$. For this reason the variables $\mathbf{w}^{(i)}$, for $i \geqq 2$ and $\Gamma$ will be called perp-space variables and spatial variations in their values will be called perp-space fluctuations. Note that not all perp-space variables correspond to phason degrees of freedom (continuous symmetries of the free energy for a generic $Q$-fold symmetric state [13]). In particular, variations of $\Gamma$ are not phasons, nor are variations in $\mathbf{w}^{(j)}$ if $j$ and $Q$ are not relatively prime. If $Q$ is a prime, however, then long-wavelength spatial variations in any of the $\mathbf{w}^{(i)}$ are properly called phasons.

The $i$-sleeve of an $n$-row border is the strip of smallest width bounded by two straight lines perpendicular to $\mathbf{e}_{n x i}$ that contains all the points $\mathbf{w}^{(i)}(v)$, where $v$ runs through all the vertices in the row border.

\subsection{Weak Matching Rules}

The $r$-atlas of a tiling is the set of all configurations appearing in the tiling that can be circumscribed by a circle of radius $r$ [7].

Local matching rules for a set of tiles are rules requiring that every configuration of radius $r$ or smaller composed of those tiles be a member of a specified $r$-atlas, where $r$ is finite. The distance $r$ is called the radius of the matching rules.

Weak matching rules for the constituent tiles of a $Q$-fold tiling are local matching rules with the following properties:

1. There exists an infinite space-filling tiling that satisfies the rules everywhere; 2. Let $\mathbf{W}$ be the lift of one of the vertices in any infinite tiling satisfying the rules. There exists a finite distance $d$ such that $\left\|\mathscr{P}^{\perp} \cdot(\mathbf{W}-\mathbf{R})\right\|<d$ for all $\mathbf{W}$ and some fixed $\mathbf{R}$, where $\|\mathbf{X}\|$ is the Euclidean norm of $\mathbf{X}$. In other words, weak matching rules ensure that in an infinite tiling perp-space fluctuations are bounded for all $i \neq 1$ (including $i=0$ ).

A tiling is said to support weak matching rules if and only if there exist weak matching rules for its constituent tiles which are satisfied by the tiling itself.

\subsection{The Alternation Condition}

The tiles in an $m$-row are of types $(m, m \pm i)$, where $i=1, \ldots,(Q-1) / 2$. A $Q$-fold tiling satisfies the alternation condition (AC) if and only if for every $m$-row and every $i \neq 0$, 


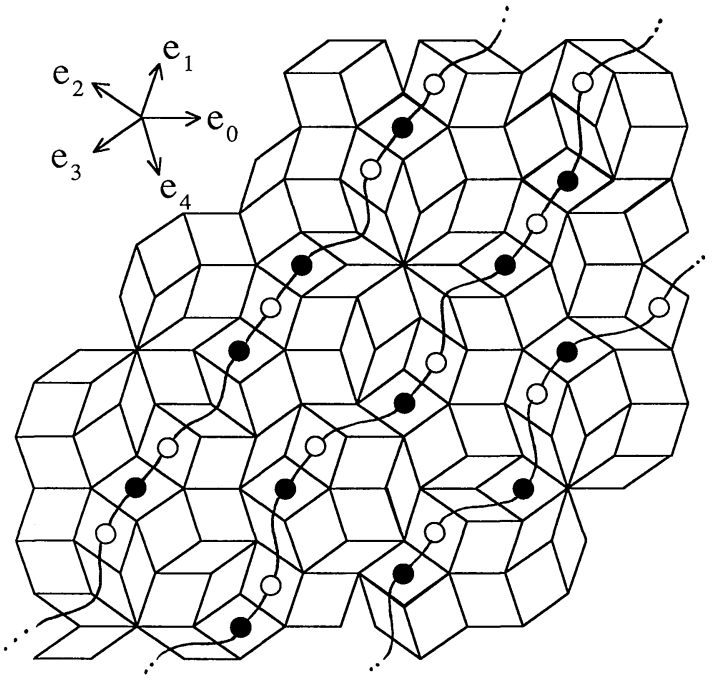

Fig. 2. Definition of the alternation condition. The star vectors are shown in the inset. $(2,3)$-tiles are marked with a solid circle. $(2,1)$-tiles are marked with an open circle. Along every 2-row the solid and open circles alternate. Note that the $(2,0)$ and $(2,4)$ tiles alternate also. The curvy lines are guides to the eye that follow the 2-rows

among the tiles lying between any two tiles of type $(m, m \pm i)$, there is a tile of type $(m, m \mp i)$. In other words, the alternation condition requires that as an $m$-row is traversed from "left" to "right" tiles of type $(m, m-i)$ and $(m, m+i)$ alternate for every $i$ (see Fig. 2). Note that the AC applies to each tile shape independently. Between two tiles of the same shape (with the same value of $i$ ) there can be any number of tiles of other shapes.

\section{Some Preliminary Results}

Lemmas 1,2 , and 3 , and Corollary 1 , below apply to $Q$-fold tilings with arbitrary odd $Q$. Lemma 4 applies only to $P$-fold tilings, where $P$ is a prime.

Lemma 1. Let $v_{1}$ and $v_{2}$ be any two vertices lying on the same m-row border in a $Q$-fold tiling obeying the AC. The value of $\left|\left(\mathbf{w}^{(i)}\left(v_{1}\right)-\mathbf{w}^{(i)}\left(v_{2}\right)\right) \cdot \mathbf{e}_{m \times i}\right|$ is uniformly bounded by $\sum_{j=1}^{(Q-1) / 2}|\cos (2 \pi i j / Q)|$.

Proof. Assume, without loss of generality, that in moving along the row border from $v_{1}$ to $v_{2}$, the edges of tiles of type $(m, m-j)$ are traversed in the $+\mathbf{e}_{m-j}$ direction. The edges of tiles of type $(m, m+j)$ will then be traversed in the $-\mathbf{e}_{m+j}$ direction. In traversing an edge in the $+\mathbf{e}_{m-j}$ direction one adds $\mathbf{e}_{(m-j) \times i}$ to $\mathbf{w}^{(i)}$, thereby increasing the value of $\mathbf{w}^{(i)} \cdot \mathbf{e}_{m \times i}$ by $\cos (2 \pi i j / Q)$. Similarly, in traversing an edge in the $-\mathbf{e}_{m+j}$ direction one adds $-\mathbf{e}_{(m+j) \times i}$ to $\mathbf{w}^{(i)}$, thereby decreasing $\mathbf{w}^{(i)} \cdot \mathbf{e}_{m \times i}$ by $\cos (2 \pi i j / Q)$. Let $n_{j \pm}$ be the number of tiles of type $(m, m \pm j)$ between $v_{1}$ and $v_{2}$. We have

$$
\left(\mathbf{w}^{(i)}\left(v_{1}\right)-\mathbf{w}^{(i)}\left(v_{2}\right)\right) \cdot \mathbf{e}_{m \times i}=\sum_{j=1}^{(Q-1) / 2}\left(n_{j-}-n_{j+}\right) \cos (2 \pi i j / Q) .
$$

The AC implies that $\left|n_{j_{-}}-n_{j+}\right|$ is either 0 or 1 , immediately implying the desired result. Q.E.D. 
Lemma 1 states that there is a bound on the width of the $i$-sleeve of any row of any type in a tiling obeying the $\mathrm{AC}$. When $Q$ is a prime, the bound is the same for all $i$ and will be referred to as $D_{s}$. When $Q$ is factorable, $D_{s}$ is taken to be the maximum value of the bound obtained for different values of $i$.

Corollary 1. Let $v_{0}$ be a vertex in a $Q$-fold tiling obeying the AC and let $v$ be any vertex on an m-row border containing $v_{0}$. Then $\mathbf{w}^{(i)}(v)$ lies within a distance $D_{s}$ of a straight line with the equation $\mathbf{w}^{(i)} \cdot \mathbf{e}_{m \times i}=\mathbf{w}^{(i)}\left(v_{0}\right) \cdot \mathbf{e}_{m \times i}$.

Proof. This follows trivially from the preceding lemma.

Lemma 2. For any two vertices $v_{1}$ and $v_{2}$ in a $Q$-fold tiling obeying the $A C$, two rows can be chosen, one having a border containing $v_{1}$, the other a border containing $v_{2}$, and the two having some tile in common.

Proof. Every vertex in a rhombic tiling lies on at least three row borders of different types, since it must be shared by at least three tiles of different types. Therefore two row borders of different types can be chosen, one containing $v_{1}$, the other $v_{2}$. The 1 -sleeves of the row borders have different slopes and therefore must intersect. Since the 1-sleeves are of bounded width (Lemma 1), the row borders contained within them must also intersect at a common vertex. The vertex belongs to a tile shared by the two rows. Q.E.D.

Lemma 3. Let $v_{1}$ and $v_{2}$ be any two vertices lying on the same m-row border in a $Q$-fold tiling obeying the $A C$ and let $\left|\left(\mathbf{w}^{(1)}\left(v_{1}\right)-\mathbf{w}^{(1)}\left(v_{2}\right)\right) \cdot \mathbf{e}_{m}^{*}\right| \equiv D$. (Recall that $\mathbf{e}_{m}^{*}$ is the rotation of $\mathbf{e}_{m}$ by $90^{\circ}$ and hence lies along the direction of the m-row.) Then $\left|\left(\mathbf{w}^{(i)}\left(v_{1}\right)-\mathbf{w}^{(i)}\left(v_{2}\right)\right) \cdot \mathbf{e}_{m \times i}^{*}\right| \equiv D^{\prime}$ is less than $\mu D$ for any $i$, where $\mu$ is the maximum value of $\sin (2 \pi j / Q) / \sin (2 \pi k / Q)$ for any integers $j$ and $k$ with $\bmod _{Q} k \neq 0$.

Proof. Assume, without loss of generality, that $\left(\mathbf{w}^{(1)}\left(v_{1}\right)-\mathbf{w}^{(1)}\left(v_{2}\right)\right) \cdot \mathbf{e}_{m}^{*}$ is positive. Each edge in the row border between $v_{1}$ and $v_{2}$ contributes $\sin (2 \pi j / Q)$ to $D$, for some $1 \leqq j \leqq(Q-1) / 2$, and $\sin (2 \pi k / Q)$ to $D^{\prime}$, where $k=i j$. Thus the absolute value of the largest possible discrepancy between the contribution of any single edge to $D$ and to $D^{\prime}$ is a factor of $\mu$. The lemma follows immediately. Q.E.D.

Lemma 4. Let $v$ be any vertex lying in a P-fold tiling obeying the AC, and let $r$ designate an m-row having $v$ on one of its borders. There exists a finite distance $D_{t}$ such that the distance between $v$ and the nearest $(m, m+i)$-tile in $r$ is less than $D_{t}$ for all $i$. (In other words, the distance from any vertex in any m-row to the nearest ( $m, m+i)$-tile is uniformly bounded.)

Proof. We will show that a tile of every type must occur within every circular region of radius $D_{t}$ in the tiling, where $D_{t}$ is not explicitly determined but is known to be finite. The basic idea is to show that a large number of tiles of some unspecified type must exist within a circle of "small" radius, then show that the presence of these tiles necessarily implies the presence of a slightly smaller number of tiles of another type within a larger radius, and finally to iterate the reasoning to show that all tile types must be represented within the large circle of radius $D_{t}$.

Consider a circle of radius $R$ (the "small" radius) and let the number of $(j, k)$ tiles contained within the circle be denoted $T_{j, k}$. Since the number of distinct tile types is finite and the number of tiles in the circle is proportional to $R^{2}$, one can 
always choose $n$ and $n^{\prime}$ such that $T_{n, n^{\prime}}$ is greater than or equal to $\pi R^{2} / A$, where $A$ is the sum of the areas of the distinct tile types. Assuming the length of a tile edge is 1 , the number of tiles in the circle that can lie on a single row is at most $2 R / W$, where $W=\sin (2 \pi / Q)$ is the width of the thinnest tile shape. Therefore the number of $n$-rows passing through the circle must be at least $T_{n, n^{\prime}} /(2 R / W) \geqq \pi W R / 2 A$. Also, the number of $n$-rows passing through the circle is at most $2 R$, so there must be at least one $n$-row that contains at least $T_{n, n^{\prime}} / 2 R \geqq \pi R / 2 A\left(n, n^{\prime}\right)$-tiles.

Let $i=n-n^{\prime}$ and $L=\pi W R / 2 A$. There is an $n$-row passing through the circle that has at least $L(n, n-i)$-tiles on it, since $W \leqq 1$. Between any two $(n, n-i)$-tiles on the same $n$-row there must be an $(n, n+i)$-tile (by the AC). Therefore, there must be at least $L-1(n+i)$-rows passing through the circle. Now Corollary 1 implies that for two $n$-rows with 1 -sleeves separated by some finite distance $d$, the presence of an $(n, n+i)$-tile in one of them implies the existence of an $(n, n+i)$-tile in the other within a distance $d \csc (2 \pi i / Q)$ along the same $(n+i)$-row, since the 1 -sleeves of the row borders must intersect at that distance. Thus a circle of radius $R \csc (2 \pi i / Q)$ (with the same center as the original circle) contains an $(L-1) \times(L-1)(n, n+i)$-net. Similarly, that same circle must contain an $(L-1) \times(L-1)(n-i, n-2 i)$-net.

Using similar reasoning applied to the $(n, n+i)$-tiles, it can be shown that there must exist an $(L-2) \times(L-2)(n+i, n+2 i)$-net (and an $(L-2) \times(L-2)(n-2 i$, $n-3 i$ )-net) within a circle of radius $R \csc ^{2}(2 \pi i / Q)$. The reasoning is then iterated to show that for a choice of $R$ such that $L$ is greater than $(Q-1) / 2$, there is at least one row of type $n+j i$ for all $j$ within a circle of radius $R \csc ^{(Q-1) / 2}(2 \pi i / Q)$. When $Q$ is a prime, this includes all star vector directions, regardless of the values of $n$ and $i$.

Thus, the distance from $v$ to the nearest row of type $m+i$ is bounded. Let $r^{\prime}$ designate this nearest row. Again, Corollary 1 implies that there is a maximum distance between the point $v$ and the intersection of the 1-sleeves of $r$ and $r^{\prime}$. Where the two sleeves intersect, there is a tile of type $(m, m+i)$. Q.E.D.

\section{The Main Result}

We are now in a position to prove that the $\mathrm{AC}$ has the properties claimed in the introduction.

First, it is easy to see that all canonical projections satisfy the AC. Recall that a canonical projection is the dual of some periodic $Q$-grid. Consider a pair of periodic grids with star vectors $m+i$ and $m-i$. These grids form a periodic lattice of rhombi. For any $i$, a grid line normal to $\mathbf{e}_{m}$ is parallel to one of the diagonals of the rhombi. The grid line must therefore intersect two adjacent edges of each rhombus that it passes through. Since these adjacent edges belong to different grids, two intersections of the same type cannot occur without the other in between. The duals of these two intersections are tiles of type $m+i$ and $m-i$.

Second, we must prove that the AC allows only uniformly bounded perp-space fluctuations.

Theorem 1. Let $v_{1}$ and $v_{2}$ be any two vertices in a $Q$-fold tiling obeying the AC. The value of $\left|\Gamma\left(v_{1}\right)-\Gamma\left(v_{2}\right)\right|$ is bounded by $Q-1$.

Proof. Applying the reasoning in the proof of Lemma 1 with $i=0$ shows that the maximum possible value of $\left|\Gamma\left(v_{3}\right)-\Gamma\left(v_{4}\right)\right|$ for $v_{3}$ and $v_{4}$ on the same row border is 
$(Q-1) / 2$. Now consider two arbitrary vertices $v_{1}$ and $v_{2}$. Let $R_{1}$ and $R_{2}$ be row borders of different types containing $v_{1}$ and $v_{2}$, respectively, and let $v_{12}$ be the vertex shared by $R_{1}$ and $R_{2}$ (Lemma 2). Writing $\Gamma\left(v_{1}\right)-\Gamma\left(v_{2}\right)=\left(\Gamma\left(v_{1}\right)-\Gamma\left(v_{12}\right)\right)$ $+\left(\Gamma\left(v_{12}\right)-\Gamma\left(v_{2}\right)\right)$ and substituting the bound $(Q-1) / 2$ twice on the right-hand side yields the desired result: $\left|\Gamma\left(v_{1}\right)-\Gamma\left(v_{2}\right)\right| \leqq Q-1$. Q.E.D.

Theorem 2. Let $v_{1}$ and $v_{2}$ be any two vertices in any $P$-fold tiling obeying the $A C$ (where $P$ is a prime number). There exists a finite number $D_{w}$ such that $\| \mathbf{w}^{(i)}\left(v_{1}\right)$ $-\mathbf{w}^{(i)}\left(v_{2}\right) \|<D_{w}$ for all $i=2, \ldots,(P-1) / 2$.

Proof. Let $v_{3}$ and $v_{4}$ be two vertices on the same $m$-row border. By Lemma 1 we already know that $\left|\left(\mathbf{w}^{(i)}\left(v_{3}\right)-\mathbf{w}^{(i)}\left(v_{4}\right)\right) \cdot \mathbf{e}_{m \times i}\right|$ is bounded. We will now prove that $\left|\left(\mathbf{w}^{(i)}\left(v_{3}\right)-\mathbf{w}^{(i)}\left(v_{4}\right)\right) \cdot \mathbf{e}_{m \times i}^{*}\right|$ is also bounded, from which the theorem will quickly follow. (Recall that $\mathbf{e}^{*}$ is the rotation of e through $90^{\circ}$.) The $P$-fold symmetry allows us to carry out the proof for the specific case of $m=0$ and from that infer its validity for all other $m$.

The proof relies on the constructions illustrated in Fig. 3. Parts (a) and (b) of the figure refer to the same sets of row borders, the difference being that (a) refers to their positions in the $\mathbf{w}^{(1)}$-plane while $(b)$ refers to the $\mathbf{w}^{(i)}$-plane.

Let $r$ be a 0 -row border containing $v_{3}$ and $v_{4}$ (lying in the strip labelled $0_{0}$ in Fig. 3a) and assume that $\left|\left(\mathbf{w}^{(i)}\left(v_{3}\right)-\mathbf{w}^{(i)}\left(v_{4}\right)\right) \cdot \mathbf{e}_{0}^{*}\right|$ equals $\Delta$ (as indicated in Fig. 3b). We will show that if $\Delta$ is chosen large enough a contradiction arises, regardless of the distance between $\mathbf{w}^{(1)}\left(v_{3}\right)$ and $\mathbf{w}^{(1)}\left(v_{4}\right)$. By Lemmas 3 and 4 , a large enough
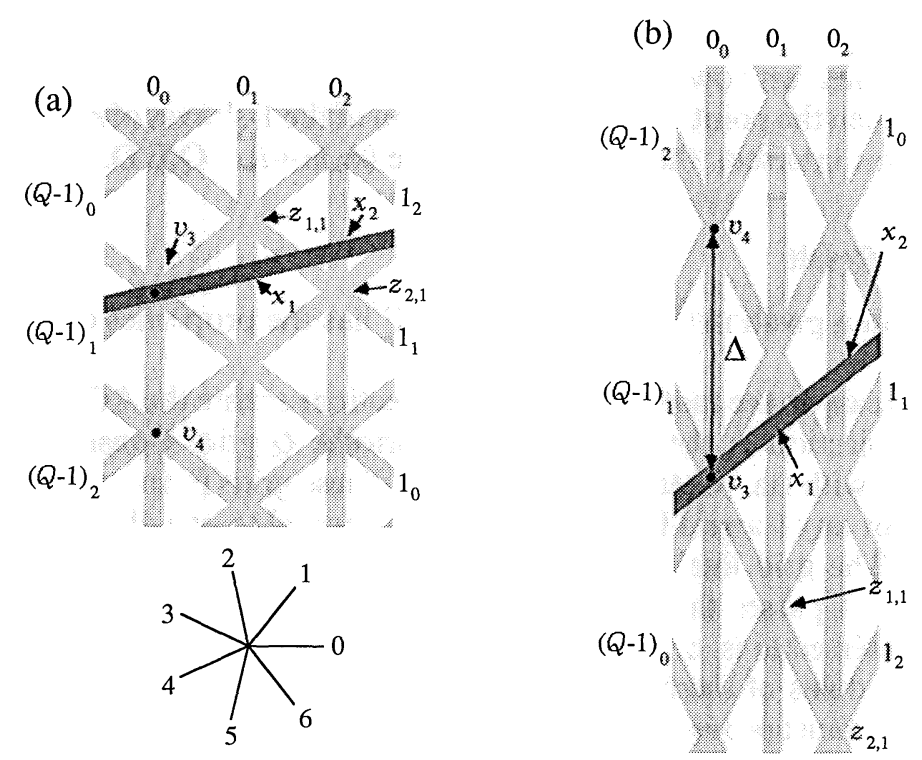

Fig. 3a, b. Proof of Theorem 2. The case chosen for the purposes of illustration is that of 7-fold symmetry. a The $\mathbf{w}^{(1)}$-plane: The shaded strips contain the 1-sleeves of the row borders used in the proof. Each strip has width $2\left(D_{t}+D_{s}\right)$ and is labelled by $\Lambda_{n}$, where $\Lambda$ specifies the star vector associated with the row border and $n$ is an integer index. The heavily shaded strip contains a 2-row. The meaning of $x_{p}$ and $z_{p, q}$ is explained in the text. b The $\mathbf{w}^{(i)}$-plane: The shaded strips contain the $i$-sleeves of the same rows labelled in a. For the illustration, we have chosen $i=3$. Strips with the same labels in $\mathbf{a}$ and $\mathbf{b}$ correspond to the same row border and $x_{p}$ and $z_{p, q}$ identify the same vertices in $\mathbf{a}$ and $\mathbf{b}$ 
choice for $\Delta$ guarantees that this distance must be larger than $2\left(D_{t}+D_{s}\right)$. Lemma 4 also implies that there exist $0-, 1-$, and $(Q-1)$-row borders in the tiling with 1 -sleeves that lie within the shaded regions of Fig. 3a, where the thickness of the shaded strips is $2\left(D_{t}+D_{s}\right)$ and the separation between parallel strips is much greater than their thickness. The shaded strips are spaced periodically to form a triangular lattice as indicated in the figure.

Corollary 1 implies that Fig. $3 \mathrm{~b}$ can be constructed such that the $i$-sleeves of the row borders lying in the shaded strips of Fig. 3a lie in shaded strips of Fig. 3b. Again, the shaded strips are spaced periodically. There are two consistent ways of labelling the strips in Fig. 3b, one of which is shown in the figure. The other is related to this one by inversion and need not be analyzed independently.

A proof that the form of Fig. $3 \mathrm{~b}$ is forced is given in the appendix. The form is a direct consequence of the facts that (1) if two vertices are connected by a finite sequence of edges their separation in the $\mathbf{w}^{(i)}$ plane is bounded (being no greater than the edge length times the number of edges in the sequence); and (2) $\Delta$, the distance between $\mathbf{w}^{(i)}\left(v_{3}\right)$ and $\mathbf{w}^{(i)}\left(v_{4}\right)$, has been chosen much larger than $D_{t}$.

Consider now the nearest 2-row border to $v_{3}$, which must lie in the heavily shaded strip in Fig. 3a [14]. Let $x_{p}$ be the vertex at the intersection of the 2-row border and the 0 -row border with index $p$. (See Fig. 3a.) Let $z_{p, q}$ be the vertex at the intersection of the 0-row border with index $p$ and the 1-row border with index $q$, where for each $p$ we choose $q$ such that $\mathbf{w}^{(1)}\left(z_{p, q}\right)$ is as close as possible to $\mathbf{w}^{(1)}\left(x_{p}\right)$. It is obvious that $\left\|\mathbf{w}^{(1)}\left(x_{p}\right)-\mathbf{w}^{(1)}\left(z_{p, q}\right)\right\|$ is bounded. On the other hand, $\| \mathbf{w}^{(i)}\left(x_{p}\right)$ $-\mathbf{w}^{(i)}\left(z_{p, q}\right) \|$ grows without bound with increasing $p$, as can be seen by the following reasoning. In the $\mathbf{w}^{(1)}$-plane the points $z_{p, q}$ lie close to a line normal to $\mathbf{e}_{2}$, implying that for large values of $p$,

$$
\left(\frac{2 q}{p}-1\right) \cot (2 \pi / Q) \Rightarrow-\cot (4 \pi / Q) \text {. }
$$

In the $\mathbf{w}^{(i)}$-plane the points $z_{p, q}$ lie close to a line with slope $\left(\frac{2 q}{p}-1\right) \cot (2 \pi i / Q)$. Substituting from Eq. (3) for $(2 q / p-1)$, we have

$$
\left(\frac{2 q}{p}-1\right) \cot (2 \pi i / Q) \Rightarrow-\cot (4 \pi / Q) \tan (2 \pi / Q) \cot (2 \pi i / Q) .
$$

But this slope cannot equal $-\cot (4 \pi i / Q)$, the slope of a line normal to $\mathbf{e}_{2 \times i}$, for any $i$ in the range $2, \ldots,(Q-1) / 2$. In the $\mathbf{w}^{(i)}$-plane, the slope of the strip containing all the $x_{p}$ is therefore different from the slope of a line approximating the $z_{p, q}$.

Thus $\left\|\mathbf{w}^{(i)}\left(x_{p}\right)-\mathbf{w}^{(i)}\left(z_{p, q}\right)\right\| /\left\|\mathbf{w}^{(1)}\left(x_{p}\right)-\mathbf{w}^{(1)}\left(z_{p, q}\right)\right\|$ grows without bound and we have a contradiction of Lemma 3 applied to the 0 -row borders of large index. The contradiction means that $\Delta$ cannot be chosen arbitrarily large and the reasoning was completely independent of the distance between $\mathbf{w}^{(i)}\left(v_{3}\right)$ and $\mathbf{w}^{(i)}\left(v_{4}\right)$. Hence $\left|\left(\mathbf{w}^{(i)}\left(v_{3}\right)-\mathbf{w}^{(i)}\left(v_{4}\right)\right) \cdot \mathbf{e}_{m \times i}^{*}\right|$ is uniformly bounded on every 0 -row border for $m=0$ in a tiling obeying the AC and, by symmetry, on every $m$-row border for arbitrary $m$. This result, along with Lemma 1, implies that $\left\|\mathbf{w}^{(i)}\left(v_{3}\right)-\mathbf{w}^{(i)}\left(v_{4}\right)\right\|<D_{w}^{\prime}$ for any two vertices on the same row border, where $D_{w}^{\prime}$ is finite.

For two vertices that are not on the same row border, Lemma 2 immediately implies $\left\|\mathbf{w}^{(i)}\left(v_{1}\right)-\mathbf{w}^{(i)}\left(v_{2}\right)\right\|<D_{w}$, where $D_{w}=2 D_{w}^{\prime}$. Q.E.D. 
We have demonstrated that the AC applied to a $P$-fold tiling allows only bounded fluctuations in $\Gamma$ and in $\mathbf{w}^{(i)}$ for all $i=2, \ldots,(P-1) / 2$. It is now easy to see that the AC can be implemented in the cases of interest using a finite $r$-atlas (the third necessary property for weak matching rules). Lemma 4 implies that there is a maximum distance between two tiles of the same type in any given row. Choosing $r$ to be greater than this distance, one merely has to include in the $r$-atlas only configurations consistent with the AC.

The main claim of this paper has now been justified: For prime values of $P$, there exist weak matching rules for P-fold symmetric tilings.

\section{Nearest Neighbor Rules for the Alternation Condition}

By allowing the tiles of the same type to be decorated in several different ways, the AC can be enforced for any $Q$-fold tiling ( $Q$ is odd) using an $r$-atlas that includes only configurations of two tiles sharing an edge. (Each distinct decoration of a configuration must be included separately in the $r$-atlas.) The construction described below produces such a decoration for any $Q$. Each tile is decorated with an arrow on each of its edges and tiles are allowed to share an edge only if their arrows on that edge are of the same type and point in the same direction. Following the discussion of the general case, the $Q=7$ cased is illustrated explicitly.

There are $(Q-1) / 2$ distinct tile shapes in a $Q$-fold tiling and we will need $Y=2^{(Q-3) / 2}$ different types of arrows. (The reason for this number will soon become clear.) For each tile shape we construct several decorations consisting of distinct ways of marking each edge with one arrow. Since an arrow can point in either of two directions and must be one of $Y$ types, the arrow on an edge represents $1+\log _{2} Y=(Q-1) / 2$ bits of information, one bit for each tile shape. The arrow on each edge can therefore be used to specify which orientation of each tile shape might be joined to that edge. Recall that a tile with two $2 \pi k / Q$ angles is called a " $k$-tile" and a tile with edges parallel to $\mathbf{e}_{i}$ and $\mathbf{e}_{j}$ is called an " $(i, j)$-tile".

For an edge parallel to $\mathbf{e}_{n}$, let $a=1$ if the arrow on it is in the $\mathbf{e}_{n}$ direction and $a=-1$ if the arrow is in the $-\mathbf{e}_{n}$ direction. Also, let the "left" and "right" sides of the edge be defined relative to the forward direction of the arrow. Now if a $k$-tile is to be added to a given edge parallel to $\mathbf{e}_{m}$, it must be either an $(m, m-k)$-tile or an $(m, m+k)$-tile. A given type of arrow specifies, for each $k$, whether a $k$-tile added to the left of the edge would have to be an $(m, m-a k)$-tile or an $(m, m+a k)$-tile. If the arrow specifies $\pm a k$ on the left, then it specifies $\mp a k$ on the right. Each arrow type specifies a particular choice of sign for each value of $k$. No two arrow types specify the same set of choices or sets that are related by the inversion of every sign. Sets related by the inversion of every sign are designated by the same type of arrow pointing in opposite directions. Opposite edges of each $k$-tile are required to have arrows that specify identical orientations for tiles of shapes other than $k$ and opposite orientations for $k$-tiles. In this way, as a tile is traversed from one edge to the opposite edge the decoration keeps track of the expected orientation of the next tile in the row according to the AC.

An example of the decoration scheme described here is shown in Fig. 4 for the case $Q=7$. Figure 4 a indicates the meaning of each type of arrow (see caption). Figure $4 \mathrm{~b}$ shows a portion of a 7 -fold tiling with tiles decorated to enforce the AC. 


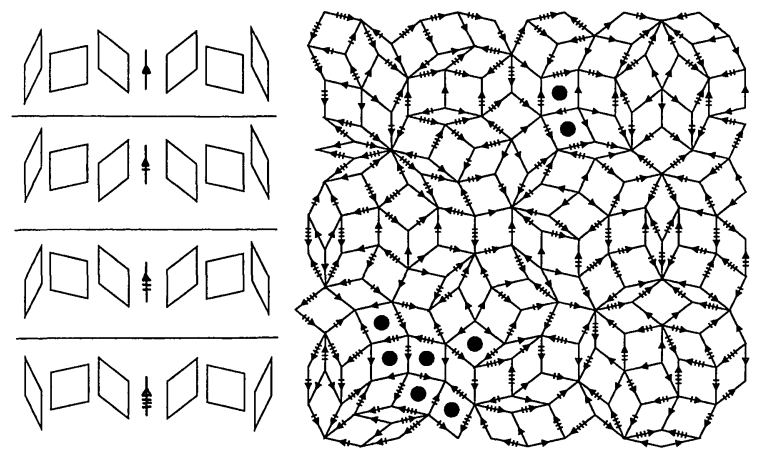

Fig. 4. A decoration that enforces the alternation condition for $Q=7$. The four types of arrows are shown at left. The three tiles to the right (left) of each arrow indicate the orientations of each tile shape that can be added to the right (left) of the arrow. (The different shapes corresporid to the different values of $k$ discussed in the text and the different orientations of corresponding tiles for different arrow types correspond to different choices of sign for a given $k$.) Note that when rotation by $180^{\circ}$ of the entire figure is taken into account, all possible sets of orientations consistent with the alternation condition are included. The decoration of a portion of a 7-fold tiling is shown at right. The eight different decorations of the 2-tile are marked with solid dots

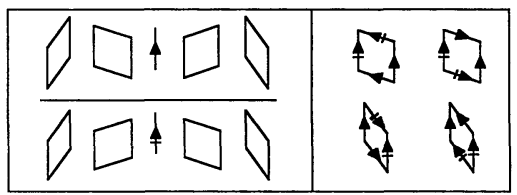

Fig. 5. A decoration that enforces the alternation condition for $Q=5$. There are two types of arrows, shown here together with the tile orientations they force on the left and right. The four tiles that are formed are shown at left

The number of distinct ways a tile of any given shape can be decorated is $Y^{2} / 2$, as determined by the following reasoning: Once a given edge is decorated the decoration on the opposite edge is determined. In addition one bit of information on each edge is fixed by the tile itself; once the arrow type for an edge of the tile is determined, the arrow direction is fixed since it is known whether the tile lies to the left or right of the edge. Thus each of two adjacent edges of the tile must be decorated in one of $Y$ ways, which would appear to produce $Y^{2}$ tile decorations. The tile decorations have been double counted, however, as they come in pairs related by rotation of the tile by $180^{\circ}$. Since there are $(Q-1) / 2$ tile shapes, the total number of distinct decorated tiles (counting enantiomorphs as distinct) is $Y^{2}(Q-1) / 4=(Q-1) 2^{Q-5}$. In Fig. 4b, the 8 different decorated 2-tiles are marked with solid circles.

Another example of a decoration scheme that is equivalent to enforcing the $\mathrm{AC}$ has already appeared in the literature. The case $Q=5$ requires four tiles which are equivalent to those discussed in ref. [9]. These are shown in Fig. 5. 


\section{An Example: 7-Fold Symmetry}

A closer examination of the decorated 7-fold tilings is worthwhile. Figure 6 shows a portion of the dual to a periodic 7-grid for which the sum of the phases, $\sum \gamma_{n}$, is an integer. It can be shown that the tiling actually has 14-fold symmetry, not just 7 -fold, and that it is locally isomorphic to all duals of periodic 7-grids in which the sum of the phases is zero. It will be referred to as a " $\Gamma=0$ tiling," where $\Gamma$ is to be thought of as the sum of the $\gamma$ 's. (The symbol " $\Gamma$ " is a natural choice since the sum of the phases of the 7-grid is proportional to $\mathscr{P}^{(0)} \mathbf{R}$, where $\mathbf{R}$ is the vector used in the definition of the corresponding canonical projection, and, as we have already seen, $\Gamma$ is used to specify positions in $E^{(0)}$.)

First, for each tile shape only seven of the eight possible decorations appear in Fig. 6. Indeed, it can be shown that the $\Gamma=07$-fold tilings do not contain any tiles of the missing types. (One shows that certain inequalities must be satisfied by the $\gamma_{n}$ in order for sequence of intersections of grid lines to correspond to a particular decoration and that these inequalities are incompatible with the condition that $\sum \gamma_{n}=0$. The proof applies to any $Q$-fold tiling, always ruling out one decoration of each tile shape.) One can therefore delete the missing decorated tiles from the original set and still have weak matching rules for the $\Gamma=0$ tilings. Equivalently, one can delete from the $r$-atlas for the undecorated tiles all configurations which would require the missing decorations and thereby obtain weak matching rules for the $\Gamma=0$ undecorated tilings. It is not known whether additional tile decorations could be deleted if one did not insist on including a canonical projection among the allowed tilings.

Second, it is interesting to examine the ways in which tiles can be rearranged without violating the matching rules. Figure 7 shows four pairs of three-tile

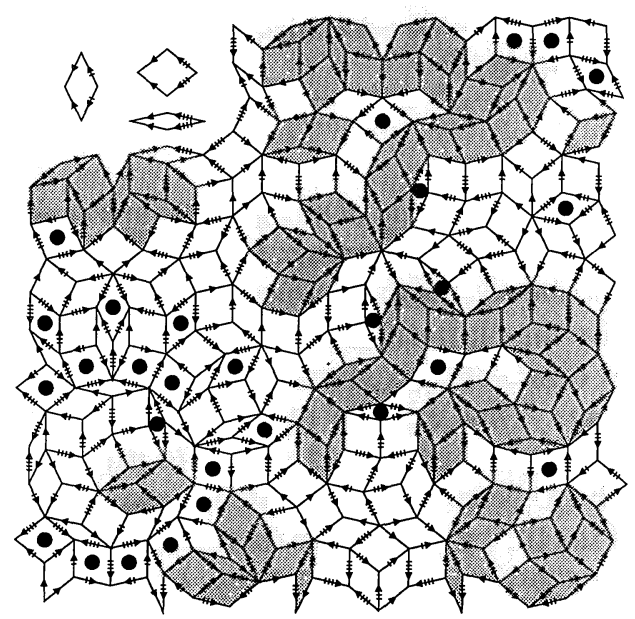

Fig. 6. A portion of a $\Gamma=07$-fold tiling. The decorated tiles shown in the inset do not occur in the $\Gamma=0$ tilings. The shaded tiles can be rearranged locally (see Fig. 7). Movement of any of the unshaded tiles necessarily involves rearrangements of infinite numbers of tiles. This is most apparent for the tiles marked with a solid dot, since their decorations are not a part of any transformable hexagon (see Fig. 7) 


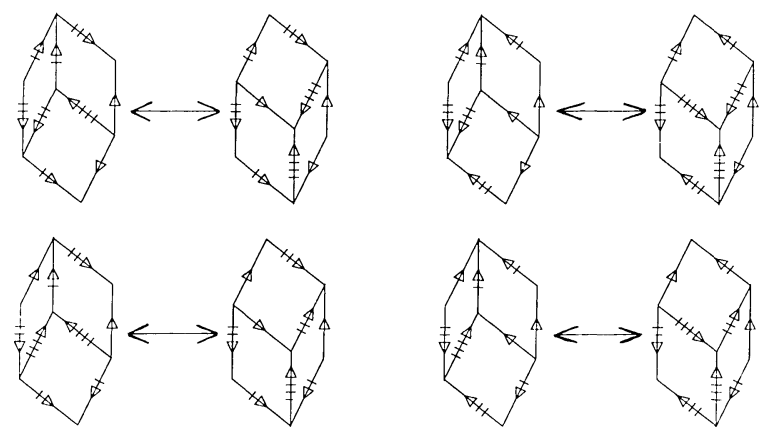

Fig. 7. Transformable hexagons of the decorated 7-fold tiling. Each member of a pair can be transformed into the other without introducing any violation of the alternation condition matching rules into the tiling. In addition to the four pairs shown here, there are four obtained from these by reflection

configurations (hexagons) that occur in any 7 -fold canonical projection (not only $\Gamma=0$ ). Either of the hexagons in a pair can be transformed into the other without changing the decoration of the exterior edges, so any member of a pair appearing in a tiling can be transformed into its partner without introducing a violation of the matching rules. The transformation of one hexagon into its partner is called a "flip." Note that a flip changes the position of the internal vertex of the hexagon and hence changes $\mathbf{w}^{(i)}$ at that vertex for all $i$. Note also that a flip can produce new hexagons in the tiling that can be flipped in turn.

The existence of transformable hexagons means that the matching rules are at best weak. For any of the shaded tiles in Fig. 6 there exists a finite sequence of flips that changes the position of the tile and therefore can introduce fluctuations in $\mathbf{w}^{(i)}$. On the other hand, there are tiles that can never participate in a flip since their decorations simply do not appear in any of the transformable hexagons. These are marked with a solid circle in Fig. 6. Loosely speaking, they give the tiling a sort of rigidity in perp-space. The perp-space variables associated with their vertices are fixed and therefore the perp-space fluctuations between them are constrained. (Note that this observation alone is not sufficient to prove that large perp-space fluctuations cannot occur in tilings that are not derived from flipping transformable hexagons in canonical projections. Nevertheless, it gives some idea of the way the matching rules function.) An important unresolved issue is whether additional decorating marks could be placed on each tile in such a way that all the hexagons are fixed and the matching rules become strong.

The flipping of transformable hexagons should not be confused with the effect of phason strains, which are long-wavelength spatial variations in the hydrodynamic variables of the system. The phason variables for a $Q$-fold tiling can be thought of as coarse grained averages of $\mathbf{w}^{(i)}$ for those values of $i$ that are relatively prime with $Q$. In the 7-fold case, coarse grained averages of $w^{(2)}$ and $w^{(3)}$ are phason variables. A small uniform shift in a phason variable induces flips of hexagons of other types than those shown in Fig. 7 and consequent transformations of the arrows on tiles that do not move at all. The presence of phason strains is signalled by isolated violations of the matching rules, just as in the familiar case of 5-fold symmetry. Details are beyond the scope of this work. 
Finally, a comparison of the 7-fold case and the well-known 5-fold case is revealing. In contrast to the 7 -fold case, the 5-fold tilings composed of decorated tiles contain no transformable hexagons. There are no hexagons that can be flipped without a consequent change in the arrows on the exterior edges, so any flip induces a violation of the matching rules. It is therefore tempting to speculate that the matching rules constructed for the 5-fold tilings actually do not allow any deviation from the canonical projections and are therefore strong matching rules, but this conjecture has yet to be proven.

The $\Gamma=0$ 5-fold tilings are just the original Penrose tilings. As in the 7-fold case, one type of decorated tile of each shape does not appear in the $\Gamma=0$ tiling. In the 5-fold case, it is known that the $\Gamma=0$ tiling supports "perfect matching rules", which restrict the possible infinite tilings to a single local isomorphism class. The effect of the analogous removal of decorated tiles in the 7-fold case is not yet understood, but the 5 -fold result indicates that it could be dramatic.

\section{Other $N$-Fold Symmetries}

\subsection{3-Fold Tilings}

The AC decoration of the tiles in a 3-fold tiling produces one type of tile: a $60^{\circ}$ rhombus with a single arrow on each edge always pointing towards the same size angle. Only one tiling consistent with the rules exists and it is periodic.

\section{2. $Q$-Fold Tilings with Factorable $Q$}

When $Q$ has two prime factors $p_{1}$ and $p_{2}$ greater than 2 , the proof of Lemma 4 breaks down. For $i$ equal to $p_{1}$ or $p_{2}$ the directions labelled $m+k i$ (modulo $Q$ ) do not include all of the star vector directions. It is easy to see the consequence: a $p_{1}$-fold or $p_{2}$-fold canonical projection is perfectly consistent with the decoration of the $Q$-fold tiles, although it uses only a subset of them. It is therefore clear that the $\mathrm{AC}$ alone is not a weak matching rule for the $Q$-fold tiling. Note that $\mathbf{w}^{\left(p_{1}+1\right)}$ is unbounded for the $p_{2}$-fold tiling constructed from a subset of the $Q$-fold tiles. Using $p_{1} p_{2}=0(\bmod Q)$ and the fact that only edges parallel to $\mathbf{e}_{p_{2} \times i}$ appear in the $p_{2}$-fold tiling, we have

$$
\begin{aligned}
\mathbf{w}^{\left(p_{1}+1\right)}(v) & =\sum_{i=0}^{p_{1}} k_{p_{2} \times i} \mathbf{e}_{\left(p_{1}+1\right) \times p_{2} \times i} \\
& =\sum_{i=0}^{p_{1}} k_{p_{2} \times i} \mathbf{e}_{p_{2} \times i} \\
& =\mathbf{w}^{(1)}(v)
\end{aligned}
$$

and $\mathbf{w}^{(1)}$ is obviously unbounded (since the tiling is infinite).

One can disallow the $p_{2}$-fold tiling simply by not including in the $r$-atlas any configurations larger than some appropriate size that do not contain at least one tile of every type, thereby making Lemma 4 true by decree. All of the other proofs carried out for prime symmetries remain valid for any odd $Q$. Thus we see that weak matching rules exist for $Q$-fold canonical projections for all odd values of $Q$. Expressing the rules as nearest neighbor matching rules for decorated tiles is much more complicated, however, when $Q$ is not a prime. 


\section{3. $N$-Fold Tilings with $N=2 Q$}

When $N$ is twice an odd number $Q$, the tilings of $N$-fold symmetry are just a subset of the $Q$-fold tilings. It can be shown that there are two classes of $Q$-fold canonical projections with $2 Q$-fold symmetry, the $\Gamma=0$ tilings and the tilings dual to periodic $Q$-grids with the sum of the phases equal to $1 / 2$. Clearly, the weak matching rules obtained by the decoration described above act as weak matching rules for the $2 Q$-fold symmetric tilings. Two interesting questions arise: First, in a physical system that is well described by the weak matching rule decoration of $Q$-fold tiles, will the equilibrium configuration have $Q$-fold symmetry or $2 Q$-fold symmetry? Second, if the appropriate decorated tiles are deleted (in analogy with the 5-fold and 7 -fold cases) is the tiling guaranteed to exhibit $2 Q$-fold symmetry? The answers are beyond the scope of this work.

\section{4. $N$-Fold Tilings with $N$ a Multiple of 4}

When $N$ is a multiple of 4 there are square tiles in the canonical projection and the AC is not well-defined. $N=4$ is a trivial case; the only tile type is a square and the only edge-to-edge tiling is periodic. For $N=8$ or $N=12$, it is known that the tiles can be decorated to yield perfect matching rules, but the construction is not as straightforward as the AC rules $[4,15]$. Higher values of $N$ have yet to be analyzed.

\section{Icosahedral Tilings}

Analogues of all of the constructions described in this paper can be defined for icosahedral tilings. The canonical projection tilings with icosahedral symmetry consist of tiles of two shapes - a prolate and an oblate rhombohedron with edges parallel to six star vectors pointing to the vertices of an icosahedron. The tile vertices are projections of $6 \mathrm{D}$ hypercubic lattice points onto a $3 \mathrm{D}$ subspace. The canonical projections can also be generated as the duals of periodic 6-grids in which the grids are sets of parallel planes normal to the star vectors. It is known that these tilings support perfect matching rules if the tiles are decorated to form 22 distinct tile types [5]. There is also strong evidence, though no rigorous proof, that perfect matching rules exist for a set of four decorated tiles - the prolate rhombohedron and the three zonohedra derived from it by the successive addition of edges parallel to the icosahedral vertex vectors [6].

The icosahedral analogue of the vectors $\mathbf{w}^{(i)}$ are the two 3D vectors $\mathbf{u}=\sum_{n=0}^{5} k_{n} \mathbf{e}_{n}$ and $\mathbf{w}=\sum_{n=0}^{5} k_{n} \overline{\mathbf{e}}_{n}$, where $\mathbf{e}_{n}$ are the icosahedral star vectors:

$$
\begin{aligned}
& \mathbf{e}_{n}=\frac{1}{\sqrt{5}}(2 \cos (2 \pi n / 5), 2 \sin (2 \pi n / 5), 1) \quad n=0, \ldots, 4 ; \\
& \mathbf{e}_{5}=(0,0,1)
\end{aligned}
$$

and $\overline{\mathbf{e}}_{n}$ are a permuted set of the star vectors:

$$
\begin{aligned}
\overline{\mathbf{e}}_{n} & =\mathbf{e}_{\bmod _{5}(2 n)} \quad n=0, \ldots, 4 ; \\
\mathbf{e}_{5} & =-\mathbf{e}_{5} .
\end{aligned}
$$




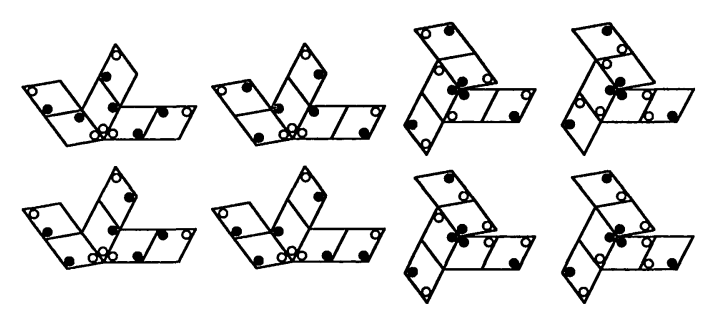

Fig. 8. The AC decoration of the rhombohedral tiles in the icosahedral canonical projections. The four shapes on the left (right) can be cut out and folded to form decorated prolate (oblate) rhombohedra. The four shapes on the top form decorated rhombohedra with a three-fold axis of symmetry, decorations which never appear in the icosahedral canonical projections

The position of a vertex $v$ in the ordinary physical space is specified by $\mathbf{u}(v)$ and its position in the complementary 3D space is specified by $\mathbf{w}(v)$. There is no analogue of $\Gamma$ for the icosahedral projections from 6D.

It is also possible to define an analogue of the alternation condition for the two rhombohedra of the canonical projection tiling. A row is now a series of rhombohedra joined along parallel faces, or, in other words, the rhombohedra dual to the intersections of each of four sets of grid planes with the line of intersection of two planes taken from the remaining two grids. The four sets of grid planes form two pairs that produce tiles of the same shape in different orientations and these alternate along the row. Using a construction similar in spirit to the edge-arrow decoration described above, a decoration that enforces the $\mathrm{AC}$ can be produced that requires 4 tile types of each shape. Figure 8 shows the eight distinct decorated tiles. The rule is that on adjoining faces both the black and the white dots must match. To see how the dot positions are determined, imagine the faces of a rhombohedron to be transparent and view the rhombohedron along a line of sight parallel to four of its edges. From this view, one sees two opposite faces overlayed. For the prolate rhombohedron, the black dot on the front face overlays the black dot on the back, while the white dots lie in opposite corners. For the oblate rhombohedron, the white dots are in the same corner and the black dots in opposite corners. The black and white dots then ensure alternation of the oblate and prolate rhombohedra, respectively. Note that four of the decorations respect the three-fold symmetry of the rhombohedra and the other four do not.

Theorem 3. The AC decoration defines a weak matching rule for the icosahedral canonical projections.

Proof. We wish to show that fluctuations in $\mathbf{w}$ are bounded. Let a set of tiles whose dual intersections lie on a single grid plane be called a "slab" and the vertices on one surface of the slab be called a "slab border" in analogy with the definitions of "row" and "row border." (Each slab border in an icosahedral canonical projection is an example of a "Wieringa roof" [3].) Consider a slab border corresponding to a grid plane with star vector $\mathbf{e}_{5}$. The projection of the slab border onto the $x y$-plane is a 5-fold tiling. Now it is straightforward to check that the AC defined on the rhombohedra acts on the projected 5-fold tiles precisely as the 2D AC studied above. Also, for a vertex $v$ on a slab border normal to $\mathbf{e}_{5}$, the $x$ and $y$ components of $\mathbf{u}(v)$ and $\mathbf{w}(v)$ are equivalent to $\mathbf{w}^{(1)}$ and $\mathbf{w}^{(2)}$ of the 5 -fold tiling, respectively. Finally, 
the $z$ components of $\mathbf{u}(v)$ and $\mathbf{w}(v)$ are equivalent to $+\Gamma$ and $-\Gamma$, respectively, where $\Gamma$ is the usual $\sum k_{n}$. Theorems 1 and 2 therefore imply that for two vertices on any slab with star vector $\mathbf{e}_{5}$ the quantity $\left\|\mathbf{w}\left(v_{1}\right)-\mathbf{w}\left(v_{2}\right)\right\|$ is bounded. Icosahedral symmetry then implies that $\left\|\mathbf{w}\left(v_{1}\right)-\mathbf{w}\left(v_{2}\right)\right\|$ is bounded on any slab. Now for any two vertices in the tiling one can choose two intersecting slabs with each of the vertices lying on one of the slabs. (Compare Lemma 2.) Since the slab borders must have the same value of $\mathbf{w}$ at their intersection, it is clear that the total variation in $\mathbf{w}$ is bounded. Q.E.D.

The icosahedral canonical projections do not contain any of the three-fold symmetric decorations shown in Fig. 8. This is simply a consequence of the fact that all of the grid planes in the dual are flat and normal to the icosahedral star vectors. (The proof is left to the reader.) Thus the three-fold symmetric decorations can be deleted from the set of allowed configurations and consistency with the canonical projections is retained. The remaining decorations are the four shown on the bottom in Fig. 8. It is interesting to note that they are equivalent to the decorations presented on p. 22 of ref. [1]. There are no analogues of transformable hexagons that can be formed by these decorated tiles and it is believed that the decoration provides perfect matching rules for the icosahedral projection tiling, but no proof has been given.

\section{Conclusion}

We have shown that tilings with $N$-fold symmetry, where $N$ is a prime or twice a prime, support weak matching rules and indicated how to construct a set of decorated tiles that serves to implement such rules. Though the result is not contradicted by any previous work, there has been no prior suggestion of its possibility. A more thorough investigation of the structure of the tilings with these symmetries may now be within reach. Just as the matching rules for Penrose tilings are intimately related to decorations that reveal the Fibonacci sequences underlying the tilings [16], the weak matching rules for other symmetries may help to uncover the relevant algebraic structures for cases in which the incommensurate ratios involved are not quadratic.

The result also revives the possibility that physical systems governed by local interactions can exhibit quasicrystalline symmetries other than those based on quadratic irrationals. More careful studies of this matter are necessary, however. For example, the ground state of a solid must correspond to a single class of locally isomorphic tilings, but weak matching rules do not select a single class. Longer range interactions or small differences in the energies of different configurations obeying the weak matching rules must become important and their possible effects should be explored. Also, the consequences of weak matching rules for elastic and hydrodynamic properties have yet to be worked out.

Finally, the fact that icosahedral tilings can support weak matching rules should be explored further. The possibility that perfect matching rules can be relaxed in certain ways but still be sufficiently restrictive to limit perp-space fluctuations means that a new variety of decoration schemes may become available for use as templates for an atomic structure. In searching for matching rules that can be implemented in a natural way in physical systems, one now has a wider range of models available. 
Acknowledgements. It is a pleasure to acknowledge useful conversations with $\mathrm{K}$. Ingersent, A. Katz, L. Levitov, P. Steinhardt and to thank D. Wright for reviewing the manuscript. The proof of Theorem 1, was formulated in a conversation with L. Tang. The significance of the alternation condition for icosahedral tilings was first suggested to me by R. Ammann. Support for this work was provided by the Harvard Society of Fellows and the NSF through grant No. DMR-88-17291.

\section{Appendix}

We wish to show that the $i$-sleeves of the row borders lying in the shaded strips of Fig. 3a must lie in the shaded regions of Fig. 3b.

First, we make repeated use of Lemma 3 to show that the strips containing the $i$-sleeves form a triangular lattice, without yet worrying about whether the strips in each direction are equally spaced. Consider the regions where three shaded strips intersect in Fig. 3a. The tiles at the intersections of the rows contained in the strips are obviously all separated by a finite distance less than or of the order of the strip width, $2\left(D_{t}+D_{s}\right)$. Lemma 3 therefore implies that the separation between the vertices of these three tiles in the $\mathbf{w}^{(i)}$ plane is bounded by some finite distance $D$. Note that $D$ depends only on $D_{t}, D_{s}$, and $i$; it is independent of $\Delta$.

Begin now with the strips labelled $0_{0}, 1_{0}$, and $(Q-1)_{1}$ and take $\Delta$ to be much larger than $D$. Consistency with Lemma 3 requires that the strip $0_{1}$ must be added and that the spacing between $0_{0}$ and $0_{1}$ is roughly equal to $(\Delta / 2) \tan (2 \pi / Q)$, the deviation being of the order of $D_{t}+D_{s}$. Applying this reasoning to strips in the other directions and then to the new triangles formed as strips are added yields a triangular lattice.

Second, similar reasoning applied to larger triangles, such as the one formed by $0_{0}, 1_{0}$, and $(Q-1)_{n}$, shows that the spacing between any two strips in a given direction whose indices differ by $n$ must be the same to within a number of the order of $D_{t}+D_{s}$. For example, consider the triangle formed by $0_{0}, 1_{0}$, and $(Q-1)_{n}$ and that formed by $0_{0}, 1_{1}$, and $(Q-1)_{n+1}$. Each of these determines a position for the strip $0_{n}$. Since the two determinations must be consistent and the strip $1_{-n}$ must intersect the intersection of $0_{0}$ and $(Q-1)_{n}$, the spacings between $1_{-n}$ and $1_{0}$ and between $1_{-n-1}$ and $1_{-1}$ must be approximately the same. Similar reasoning applies to the other directions.

Considering the sequence of positions $y_{n}$ of the centers of the strips normal to a given direction, we have just shown that

$$
y_{m}-y_{m-n}=s_{n}+\varepsilon_{m, n},
$$

where $s_{n}$ is a constant (independent of $m$ ) of the order of $\Delta$ and $\varepsilon_{m, n}$ is of the order of $D_{t}+D_{s}$. In order to satisfy the equation for all $n$ simultaneously, we must have

$$
y_{m}=m s+\varepsilon_{m},
$$

where $s$ is a constant depending only on the direction of the strip and $\varepsilon_{m}$ is bounded by a number on the order of $D_{t}+D_{s}$. The strips can be enlarged so that when equally spaced they include the possible deviations in $\varepsilon_{m}$. There is no danger of parallel strips overlapping since $s$ is of the order of $\Delta$, which can be chosen as large as desired. Symmetry immediately guarantees that $s$ is the same for the strips in the 1 and $(Q-1)$ directions. Hence, the triangular lattice of strips can always be made to have the form shown in Fig. 3b. 


\section{References}

1. Steinhardt, P.J., Ostlund, S.: The physics of quasicrystals. Singapore: World Scientific 1987

2. Penrose, R.: Bull. Inst. Math. Appl. 10, 266 (1974)

3. de Bruijn, N.: Proc. Kon. Nederl. Akad. Wetensch. A 84, 39 (1981)

4. Socolar, J.E.S.: Phys. Rev. B 39, 10519 (1989)

5. Katz, A.: Commun. Math. Phys. 118, 263 (1988)

6. Socolar, J.E.S., Steinhardt, P.J.: Phys. Rev. B 34, 617 (1986)

7. Levitov, L.S.: Commun. Math. Phys. 119, 627 (1988)

8. Socolar, J.E.S.: In Quasicrystals - Adriatico Anniversary Research Conference. Jaric, M.V., Lundqvist, S. (eds.). Singapore: World Scientific (to appear) (1989)

9. Kleman, M., Pavlovitch, A.: J. Phys. C 3, Workshop on Aperiodic Crystals: 229 (1986)

10. Elser, V.: J. Phys. A. Math. Gen. 17, 1509 (1984)

11. Henley, C.L.: J. Phys. A. Math. Gen. 21, 1649 (1988)

12. Gahler, F., Rhyner, J.: J. Math. Phys. A 19, 267 (1986)

13. Lubensky, T.C.: In Aperiodicity and Order, vol. 1, Jaric, M.V. (ed.). Boston: Academic Press 1988

14. One could equally well consider a $c i$-row border, where $c$ is any number relatively prime to $Q$, but only one case is necessary for the proof

15. Katz, A.: Unpublished

16. Levine, D., Steinhardt, P.J.: Phys. Rev. B 34, 596 (1986)

Communicated by A. Jaffe

Received September 28, 1989 
